(NASA-CR-200038) GRAVITY WAVE

MOMENTUM FLUX IN THE LOWER

STRATOSPHERE OVER CONVECTION

(NASA. Ames Research Center)
$4 p$

Unclas 


\section{Gravity wave momentum flux in the lower stratosphere over convection}

\author{
M. Joan Alexander \\ University of Washington, Seattle, Washington
}

Leonhard Pfister

NASA Ames Research Center, Moffett Field, California

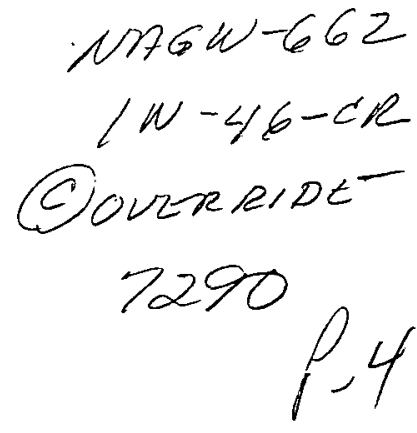

Abstract. This work describes a method for estimating vertical fluxes of horizontal momentum carried by short horizontal scale gravity waves $\left(\lambda_{x}=10-100\right.$ $\mathrm{km}$ ) using aircraft measured winds in the lower stratosphere. We utilize the in situ wind vector and pressure altitude measurements provided by the Meteorological Measurement System (MMS) on board the ER-2 aircraft to compute the momentum flux vectors at the flight level above deep convection during the tropical experiment of the Stratosphere Troposphere Exchange Project (STEP-Tropical). Data from Flight 9 are presented here for illustration. The vertical flux of horizontal momentum in these observations points in opposite directions on either side of the location of a strong convective updraft in the cloud shield. This property of internal gravity waves propagating from a central source compares favorably with previously described model results.

\section{Introduction}

The evolving picture of how tropospheric wave sources control the structure and dynamics of the middle atmosphere now includes the important role played by high frequency gravity waves. Although typical power spectra of horizontal velocity or temperature perturbations are red in frequency, suggesting that the low frequency waves hold most of the energy, the high frequency waves carry a disproportionate share of the gravity wave momentum flux. This is particularly true in the mesosphere, where Fritts and Vincent [1987] found twothirds of the momentum flux was carried by the highest frequency band they observed: waves with periods between 8 minutes and an hour. It is the important quantity of momentum flux (and its divergence) that describes the mean flow acceleration due to these waves, which may be driving the middle atmosphere away from its radiatively determined state, and may play a role in forcing the semiannual oscillation (SAO) in the tropical mesosphere. (See e.g. Andrews et al. [1987] for details and references.) There is in general a lack of information about these shorter gravity waves which are rela-

\section{Copyright 1995 by the American Geophysical Union.}

Paper number 95GL01984

0094-8534/95/95GL-01984\$03.00 tively difficult to observe. Few details of the spatial or temporal distribution or strength of their sources have been determined, and so they cannot adequately be described in global circulation models or in studies of the SAO.

A likely source for high frequency gravity waves is convection. Alexander et al. [1995] describe observational evidence for high frequency waves associated with convection, and describe the spectral properties of stratospheric gravity waves appearing above the deep convection in their simulation of a two-dimensional midlatitude squall line. The stratospheric waves in their simulation had horizontal scales in the $10-100 \mathrm{~km}$ range, and intrinsic periods between 6 minutes and 1 hour. They also note that the change in direction of gravity wave momentum flux ahead of and behind the storm center may serve as a signature of waves generated by this source (Figure 1). This signature should be present in the flux carried by plane waves close to a concentrated source such as a convective updraft because for upward conservative wave propagation, the ratio of momentum flux to horizontal group velocity relative to the source is positive if the shear is not too strong.

Pfister et al. [1993] describe some of the advantages in using ER-2 data for observing mesoscale waves generated by convection. In this paper, we develop a statistical approach to the analysis of data from the Meteorological Measurement System (MMS) [Scott et al., 1990] to identify features of waves with scales $\leq 100 \mathrm{~km}$ as an ensemble. Waves of this scale demand a statistical ensemble approach because they tend to be individually smaller in amplitude and in wave packet size, yet are ubiquitous, and so collectively may have profound effects on the middle atmosphere. We describe a method for estimating momentum flux carried by $10-100 \mathrm{~km}$ scale waves along the ER-2 flight track, and compare the results to the Alexander et al. [1995] simulation. A further comparison of ozone and temperature measurements provides a consistency check on the interpretation of the perturbations as due to gravity wave vertical parcel displacements.

\section{MMS Flight Data Analysis}

\subsection{Momentum Flux}

Gravity wave momentum fluxes are computed from MMS wind vectors $(u, v, w)$ after application of a 10-100 


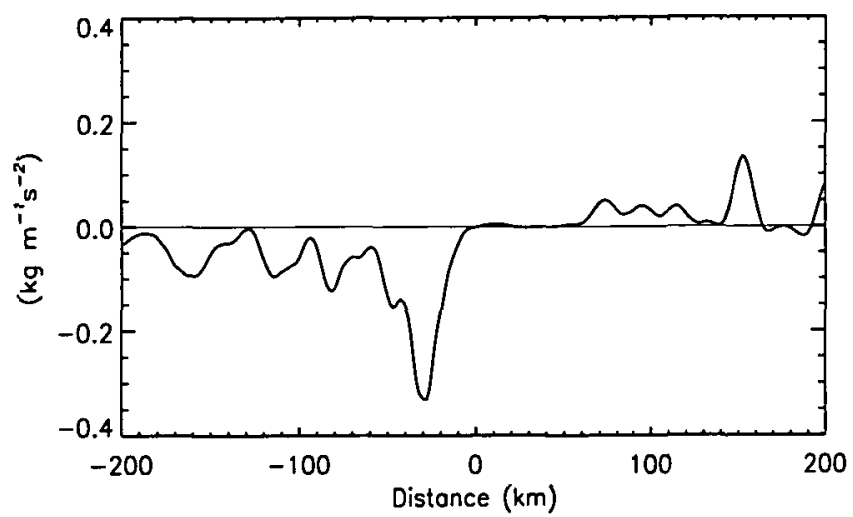

Figure 1. Vertical flux of horizontal momentum, $p_{0} u^{\prime} w^{\prime}$ as a function of distance from storm center on the $75 \mathrm{hPa}$ pressure surface $(16 \mathrm{~km})$. These fluxes are derived from the two-dimensional squall line simulation in Alexander et al. [1995], and should be similar to those observed along an ER-2 flight path over a strong convective point source. The plane waves that carry the flux, point to their source at $x=0$ via the change in sign from left to right of storm center. The flux has been smoothed for comparison to the data shown in Figure 2.

$\mathrm{km}$ band-pass filter to the Fourier decomposed fields. The filter is a smoothed version of an ideal band-pass [Percival and Walden, 1993] that removes mean flow and large scale wind variations, including most inertiogravity wave perturbations, as well as small scale fluctuations that are more likely contaminated with noise and turbulent air motions. Filtered winds $\left(u^{\prime}, v^{\prime}, w^{\prime}\right)$ are then perturbations at the $10-100 \mathrm{~km}$ scales. From these, vertical flux of horizontal momentum $\rho_{0}\left(u^{\prime} w^{\prime}, v^{\prime} w^{\prime}\right)$ is computed and smoothed with a $10-\mathrm{km}$ running triangular smoothing function. Note that the flux is not horizontally averaged over wavelength so phase variations will be evident in the result.

Time along the flight path has been converted to distance via the aircraft speed. The Fourier analysis assumes that the wave pattern is stationary along the flight leg. Flying at $200 \mathrm{~m} \mathrm{~s}^{-1}$, the plane travels 400 $\mathrm{km}$ in $33 \mathrm{~min}$. Violations of the stationarity assumption may affect the long wavelength cutoff of the bandpass filter but will not affect the velocity correlations because the aircraft speed is much larger than the wave phase speeds.

Data from Flight 9 of the STEP-Tropical experiment [Russell et al., 1993] is chosen to illustrate the technique. The initial overpass (at $18 \mathrm{~km}, 75 \mathrm{hPa}$ ) crosses a roughly linear cloud band. The aircraft data report a steady flight altitude along the straight $400 \mathrm{~km}$ long leg from $11^{\circ} \mathrm{S} 129.5^{\circ} \mathrm{E}$ to $8^{\circ} \mathrm{S} 139^{\circ} \mathrm{E}$ (see Figure 2) that runs perpendicular to the cloud band. Following this high altitude leg, the inertial navigation system failed, so we have no MMS data for subsequent passes over this cloud shield.

The momentum flux vectors along this flight path are shown in Figure 2. Maximum values are $0.12 \mathrm{~kg} \mathrm{~m}^{-1} \mathrm{~s}^{-2}$ and northeastward in direction at $10.4^{\circ} \mathrm{S}$ latitude. The momentum flux can be seen to point in the opposite

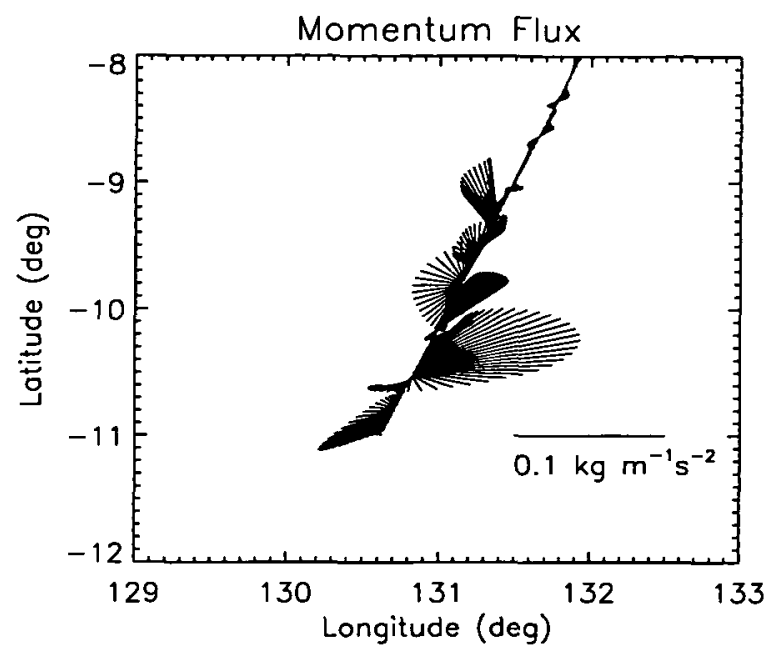

Figure 2. Momentum flux vectors, $\rho_{0}\left(u^{\prime} w^{\prime}, v^{\prime} w^{\prime}\right)$ along the flight path shown as a function of latitude and longitude. Each vector begins at the point of measurement on the flight path (the line running south-southwest to north-northeast). The maximum values point away from a region of very active convection at $10.5^{\circ} \mathrm{S}$.

direction just south of this position. The flux points to the northeast throughout the $60 \mathrm{~km}$ north of $10.5^{\circ} \mathrm{S}$, and to the southwest over most of the $60 \mathrm{~km}$ south of this position. This change in the direction of momentum flux occurs where the coldest, highest clouds appear below the aircraft. The apparent rotary character of the flux vectors is caused by smoothing effects where the flux direction changes sharply, and is not likely to be physically meaningful.

We have no infrared cloud brightness temperature measurements on the $18 \mathrm{~km} \mathrm{leg}$, however, the aircraft turned and retraced its path on a $15.2 \mathrm{~km}$ leg crossing the same latitude $48 \mathrm{~min}$ later. (Note that the mean wind is perpendicular to the flight track and parallel to the cloud band which is at an approximately constant latitude during this time.) Brightness temperature in the $10.6 \mu \mathrm{m}$ channel [Liou et al., 1990] of the $15.2 \mathrm{~km}$ leg is shown in Figure 3. On a subsequent 16.4 $\mathrm{km} \mathrm{leg}$, the pilot had to climb to avoid flying through

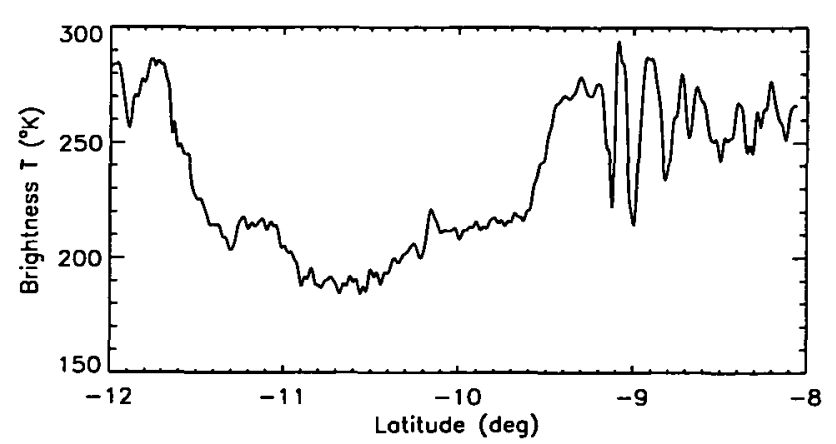

Figure 3. Brightness temperature along the flight track (shown as a function of latitude) from the downward looking narrow field of view radiometer $10.6 \mu \mathrm{m}$ channel. The lowest temperatures show the location of the coldest highest cloud tops, and indicate the region of deepest convection. 
an overshooting cloud turret that appeared at $10.5^{\circ} \mathrm{S}$ $10.4^{\circ} \mathrm{S}$, an indication of strong vertical motions within the cloud below this position, and hence a likely source of gravity waves. We conclude the change in direction of momentum flux either side of this position points to a source below. Further to the north, fluxes generally decrease but are northward in direction, pointing away from the highest clouds, consistent with the source for these wave motions in the deep convection just to the south. Even further north along the flight path, the root mean square (rms) magnitude of the flux decreases by a factor of 4 after the aircraft passes beyond the edge of the high cloud band at $\sim 9.3^{\circ} \mathrm{S}$. This sharp transition is further indication that the flux is carried by high frequency waves which propagate along more vertically oriented rays than the lower frequency inertio-gravity waves.

\subsection{Coherency of Ozone and Temperature Mea- surements}

Simultaneous ozone mixing ratio $\left(X_{\mathrm{O}_{3}}\right)$ measurements along the flight path [Proffitt et al., 1989] show a very high degree of correlation with temperature (T) observations by the MMS, a property noted by Pfister et al. [1986] in a similar set of measurements. The Flight 9 band-pass filtered data show this coherency quite clearly. (Compare Figure 4a with solid line in 4b.) This high degree of coherency provides an opportunity to test the interpretation of the observed velocity perturbations as due to gravity waves. Any errors in the aircraft altitude would in turn indicate errors in the vertical velocity and vertical momentum flux because the winds are determined as the small residual difference of two large vector quantities: the ground-relative aircraft velocity, and the aircraft-relative wind velocity [Scott et al., 1990]. The reported altitude in these measurements
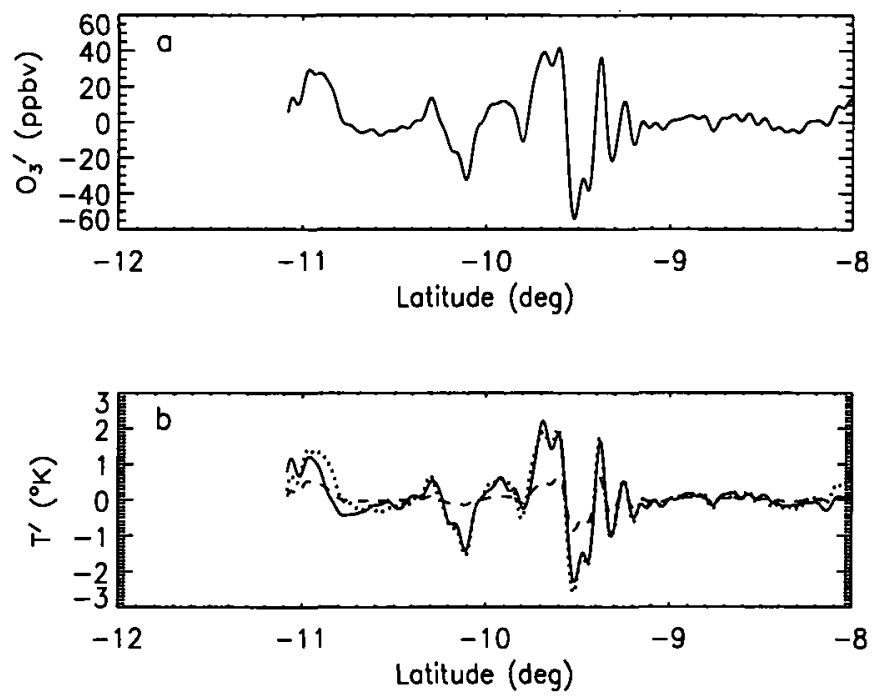

Figure 4. Perturbations along the flight track in (a) ozone mixing ratio and (b) temperature, shown as a function of latitude. In (b) the solid line is $T^{\prime}$ measured by the MMS, while the dashed and dotted lines show the results of Eqns. 1 and 2 respectively. is pressure altitude, $z_{p}=H \ln \left(p_{0} / p\right)$, where $H$ is the scale height, $p$ is pressure, and $p_{0}$ is surface pressure. In the convection simulation described in the introduction, variations in $z_{p}$ are $\sim \pm 10-20 \mathrm{~m}$ along the constant geometric altitude surface in Figure 1. These are the same magnitude as variations in $z_{p}$ reported along the flight $9 \mathrm{leg}$, so we treat geometric altitude along this leg as constant within $\sim \pm 10-20 \mathrm{~m}$.

The coherency between $X_{O_{3}}$ and T perturbations could be explained as either due to vertical adiabatic parcel displacements or aircraft vertical motions, since at this altitude $(18 \mathrm{~km})$ both $X_{O_{3}}$ and $\mathrm{T}$ have positive vertical gradients in their mean state profiles. However, the relative amplitudes in the perturbations $X_{O_{3}}^{\prime}$ and $\mathrm{T}^{\prime}$ differ in these two scenarios: The parcel displacement model includes the effects of adiabatic heating and cooling on $T^{\prime}$, while the aircraft motion model would sense only the vertical gradients.

We estimate the vertical displacement $\Delta z^{\prime}$ associated with the observed $X_{O_{3}}^{\prime}$ via:

$$
\Delta z^{\prime}=-X_{O_{3}}^{\prime} \cdot\left(\frac{\partial \bar{X}_{O_{3}}}{\partial z}\right)^{-1}
$$

where $\partial \bar{X}_{O_{3}} / \partial z$ is the gradient in the background ozone mixing ratio. Vertical displacements derived in this manner for Flight 9 are $\sim \pm 50-200 \mathrm{~m}$, an order of magnitude larger than the variations in the reported pressure altitude variations.

Two temperature perturbation models are then computed from these $\Delta z^{\prime}$ :

(1) Assume $\Delta z^{\prime}$ are changes in aircraft altitude.

$$
\mathrm{T}_{A C}^{\prime}=-\Delta z^{\prime} \cdot \frac{\partial \overline{\mathrm{T}}}{\partial z}
$$

(2) Assume $\Delta z^{\prime}$ are vertical gravity wave parcel displacements.

$$
\mathrm{T}_{G W}^{\prime}=-\Delta z^{\prime} \cdot\left(\frac{\partial \overline{\mathrm{T}}}{\partial z}+\frac{g}{c_{p}}\right)
$$

$\partial \overline{\mathrm{T}} / \partial z$ is the gradient in the mean state $\mathrm{T}$ field, and $g / c_{p}$ is the adiabatic lapse rate. The mean state temperature gradient is taken from the microwave temperature profiler observations [Gary, 1989] smoothed to capture only the large scale variations. $\partial \overline{\mathrm{T}} / \partial z$ varies between 1 and $5.5^{\circ} \mathrm{K} \mathrm{km}^{-1}$ along the flight path. Potential temperature gradient $\partial \bar{\theta} / \partial z$ is easily obtained from $\partial \overline{\mathrm{T}} / \partial z$. The ozone vertical gradient is then assumed proportional to $\partial \bar{\theta} / \partial z$ via the ratio of the ozone and potential temperature gradients determined from the aircraft ascent profiles (linear fit above $16.6 \mathrm{~km}$ ). $\partial \bar{X}_{O_{3}} / \partial z$ varies between 220 and 320 ppbv km${ }^{-1}$ along the horizontal flight leg.

Comparison of the models described by Eqns. 1 and 2 above and the observed temperature perturbations are shown in Figure 4b as the dashed and dotted lines, respectively. The vertical parcel displacement model, $\mathrm{T}_{G W}^{\prime}$ (dotted line), provides a much better fit to the 
observed temperature perturbations, and suggests gravity wave vertical parcel displacements of $\sim 50-200 \mathrm{~m}$. The standard error estimate describing the difference between Eqn. 2 and the observed $\mathrm{T}^{\mathrm{v}}$ is $0.2^{\circ} \mathrm{K}$, which is less than the accuracy in the observations [Scott et al., 1990]. Other errors in this type of analysis could arise from errors in the vertical ozone and temperature gradients. Accuracy in $\partial \bar{T} / \partial z$ is reported as $10 \%$ [Gary, 1989]. Errors in the ozone gradient are expected to be comparable.

It is also noteworthy that the rms values of perturbations in both ozone and temperature decrease suddenly by factors of $4-5$ north of $\sim 9.3^{\circ} \mathrm{S}$, just as the momentum flux decreased at this same location. As can be seen in Figure 3, this location corresponds roughly to the edge of the high cloud band.

\section{Discussion and Conclusions}

The observed momentum fluxes in Figure 2 qualitatively compare favorably with the theoretical model results shown in Figure 1 . We see the momentum flux point in opposite directions on either side of a likely source region. The observed fluxes are, however, smaller by a factor of three than those in the 2-d model. This might be expected for any of a number of reasons: The 2-d model might simply overpredict amplitudes in 3-d; the flight path may not sample the region of highest gravity wave flux; shear in the real lower stratosphere can limit wave propagation; and partial cancelation via superposition of waves carrying flux of opposite sign can limit observed net magnitudes. The model is a highly idealized, 2-d, midlatitude storm. Simulations with more similar conditions to the observations might best be compared to a statistical sample of fluxes over convection observed with the MMS on the ER-2 in future work.

Amplitudes of perturbations in the ozone mixing ratio and temperature measured along the flight path agree with the interpretation of these perturbations as due to gravity wave vertical parcel displacements. This may serve as an aid in interpreting the momentum fluxes determined with the method described in this paper. Large discrepancies in the $\mathrm{T}^{\mathrm{N}}-\mathrm{T}_{G W}^{\prime}$ comparison in other measurements could point to potential errors in the determination of the aircraft altitude, and hence errors in the vertical velocity and vertical flux of horizontal momentum.

The rms perturbations in momentum flux, ozone, and temperature all decrease by a factor of $4-5$ when the plane flies north of the edge of the high cloud band, compared to measurements directly over the high cloud band. This observation may be related to the likely high intrinsic frequencies of the small scale waves included in these observations. According to the gravity wave dispersion relation, such waves would propagate at fairly steep angles from the horizon, and not be detectable far from their source at the altitudes of the aircraft measurements.

Acknowledgments. This work was supported by the Physical Meteorology Program of the National Science Foundation, NSF grant ATM-9322480; and by the National Aeronautics and Space Administration, NASA Grant NAGW662.

\section{References}

Alexander, M.J., J.R. Holton, and D.R. Durran, The gravity wave response above deep convection in a squall line simulation, J. Atmos. Sci., 52, 2212-2226, 1995.

Andrews, D.G., J.R. Holton, and C.B. Leovy, Middle Atmosphere Dynamics, Academic Press, Orlando, 489 pp., 1987.

Fritts, D.C., and R.A. Vincent, Mesospheric momentum flux studies at Adelaide, Australia: Observations and a gravity wave-tidal interaction model, J. Atmos. Sci., 44, 605-619, 1987.

Gary, B.L., Observational results using the microwave temperature profiler during the airborne antarctic ozone experiment, J. Geophys. Res., 94, 11,223-11,231, 1989.

Liou, K.N., S.C. Ou, Y. Takano, F.P.J. Valero, and T.P. Ackerman, Remote sounding of the tropical cirrus cloud temperature and optical depth using 6.5 and $10.5 \mu \mathrm{m}$ radiometers during STEP, J. Appl. Meteor., 29, 716-726, 1990.

Percival, D.B., and A.T. Walden, Spectral analysis for physical applications: Multitaper and conventional univariate techniques, University Press, New York, 702 pp., 1993.

Pfister, L., W. Starr, R. Craig, M. Loewenstein, and $M$. Legg, Small-scale motions observed by aircraft in the tropical lower stratosphere: evidence for mixing and its relationship to large-scale flows, J. Atmos. Sci., 43, 3210$3225,1986$.

Pfister, L., K.R. Chan, T.P. Bui, S. Bowen, M. Legg, B. Gary, K. Kelly, M. Proffitt, and W. Starr, Gravity waves generated by a tropical cyclone during the STEP tropical field program: A case study, J. Geophys. Res., 98, 86118638, 1993.

Profitt, M.H., M.J. Steinkamp, J.A. Powell, R.J. McLaughlin, O.A. Mills, A.L. Schmeltekopf, T.L. Thompson, A.F. Tuck, T. Tyler, R.H. Winkler, and K.R. Chan, In situ ozone measurements within the 1987 Antarctic ozone hole from a high-altitude ER-2 aircraft, J. Geophys. Res., 94, $16,547-16,555,1989$.

Russel, P.B., L. Pfister, and H.B. Selkirk, The tropical experiment of the Stratosphere-Troposphere Exchange Project (STEP): Science objectives, operations, and summary findings, J. Géophys. Res., 98, 8563-8589, 1993.

Scott, S.G., T.P. Bui, K.R. Chan, and S.W. Bowen, The meteorological measurement system on the NASA ER-2 aircraft, J. Atmos. Ocean. Tech., 7, 525-540, 1990.

M. J. Alexander, University of Washington, Department of Atmospheric Sciences, AK-40, Seattle, Washington 98195

(received March 28, 1995; revised May 31, 1995; accepted June 15, 1995.) 